\title{
Boundary conditions and the entropy bound
}

\author{
Sergey N. Solodukhin* \\ Spinoza Institute, University of Utrecht, Leuvenlaan 4, 3584 CE Utrecht, The Netherlands
}

(Received 10 October 2000; published 11 January 2001)

\begin{abstract}
The entropy-to-energy bound is examined for a quantum scalar field confined to a cavity and satisfying Robin's condition on the boundary of the cavity. It is found that near certain points in the space of the parameter defining the boundary condition the lowest eigenfrequency (while nonzero) becomes arbitrarily small. Estimating, following Bekenstein and Schiffer, the ratio $S / E$ by the $\zeta$-function $(24 \zeta(4))^{1 / 4}$, we compute $\zeta(4)$ explicitly and find that it is not bounded near those points that signal violation of the bound. We interpret our results as imposing certain constraints on the value of the boundary interaction and estimate the forbidden region in the parameter space of the boundary conditions.
\end{abstract}

DOI: 10.1103/PhysRevD.63.044002

PACS number(s): 04.70.-s

\section{INTRODUCTION}

Some time ago Bekenstein proposed $[1,2]$ that for a quantum system confined to a cavity of finite size $R$ the entropy to the energy ratio $S / E$ cannot be arbitrarily large so that the bound

$$
S / E \leqslant 2 \pi R
$$

takes place. Originally the bound was deduced by considering a gedanken experiment of lowering the system into a black hole and demanding this process to satisfy the generalized second law. The bound (1) comes out as a consistency condition between black hole thermodynamics and ordinary statistical physics. Since the system initially can be placed far away from the black hole where the gravitational field is negligible, the bound must hold for any system in flat spacetime and be provable with no recourse to gravitational physics. Thus, the universality of (1) was conjectured.

The black hole way of deriving the bound was criticized in [3] (see, however, [4]). Nevertheless, the bound (1), as it stands, has passed a number of tests [1,2,5-7], (for a review see [8]) so that its universality deserves further examination (for a recent discussion see [9] and [10]). In order to make the statement on the entropy bound precise one has to define the meaning of $S, E$, and $R$ in (1) as well as the conditions under which the statistical properties of the system should be considered. In Ref. [5] the bound (1) is regarded as applying only to the field in the cavity and it is proposed to use microcanonical methods. One interprets $S$ as logarithm of $\Omega(E)$, the number of quantum states accessible to the field system with energy up to and including $E$, and ignores the walls of the cavity.

The bound (1) can obviously be exceeded if there are one-particle states with zero energy (zero modes) [11]. Then, by adding an arbitrary number of such states one does not change the total energy of the system but makes the entropy $S$ arbitrarily large. The important observation [12], however, is that the zero mode with some occupation corresponds to a condensate. The systems with different configurations of the

*Email address: S.Solodukhin@ phys.uu.nl condensate should be considered as macroscopically different. Thus, only the excitations with energy above the vacuum should be taken into account, i.e., the zero modes are to be excluded.

Suppose the cavity confining the system is circumscribed by a sphere of radius $R$. Then, it was shown in [5] that the microcanonical entropy $S(E)=\ln \Omega(E)$ obeys

$$
S(E) / E<\left[24 \zeta_{s p}(4)\right]^{1 / 4},
$$

where $\zeta_{s p}(k)$ is the $\zeta$ function

$$
\zeta(k)=\sum_{i} \omega_{i}^{-k}
$$

for the sphere, where $\left\{\omega_{i}\right\}$ is the discrete one-particle energy spectrum with zero modes excluded. Since for the sphere we have $\zeta(4) \sim R^{4}$ the bound (1) follows from (2) provided $R^{-4} \zeta(4)$ is appropriately bounded from above. The latter was verified in [5] for various types of free fields satisfying Dirichlet or Neumann conditions on the sphere.

In this paper we take a step further and impose more general conditions of Robin type on the field on the boundary of the cavity. Note that the boundary condition of this type should always be imposed on a quantum field nonminimally coupled to the metric. The simplest case is the scalar field described by the action

$$
W=-\frac{1}{2} \int_{M}\left[(\nabla \phi)^{2}+\xi \phi^{2} R\right]-\int_{\partial M} \xi \phi^{2} K,
$$

where $R$ is the Ricci scalar and $K$ is the extrinsic curvature of the boundary $\partial M$. The boundary term is necessary to add to the stress tensor in order for the theory to be well defined. Variation of this action with respect to $\phi$ gives us not only the equation of motion in the interior

$$
-\nabla^{2} \phi+\xi R \phi=0
$$

but also the boundary condition

$$
\left(n^{\mu} \partial_{\mu} \phi+2 \xi K \phi\right)_{\partial M}=0
$$


of Robin type. Note also that allowing for a more general boundary condition is in accord with the general assumption of the Schiffer-Bekenstein paper [5] that all interactions of the field are negligible "except for those which confine it and are expressed as boundary conditions." The boundary condition (3) encodes in a generic form such boundary interaction [13].

The $\zeta$ function is a simpler object for computation (the computational technique appropriate to the case under consideration was developed in [14] and [15]) than the ratio $S(E) / E$. Therefore, in this paper we mainly analyze the $\zeta$ function. Considering $R^{-4} \zeta(4)$ as a function of the parameter in the Robin boundary condition we find that there are special points in the parameter space near which this function is unbounded from above. This can be easily understood. Exactly at those special points the quantum field has a zero mode. When any of these points is approached in the parameter space it signals that the lowest eigenfrequency $\omega_{1}$, while nonzero, becomes arbitrarily small. Since $\omega_{1}$ makes the dominant contribution to the $\zeta$ function (see also [2]), one has $\zeta(4) \simeq 1 / \omega_{1}^{4}$ if the lowest energy state is nondegenerate. It is evident that $\zeta(4)$ is unbounded in this case.

The same is also true for the ratio $S(E) / E$ itself. When the parameter in the boundary condition approaches one of those special points it is typical that in the spectrum there appears a large gap between the lowest (nonzero) eigenfrequency $\omega_{1}$ and the next eigenfrequency $\omega_{2}, \omega_{2} / \omega_{1} \gg 1$. When the energy of the system is $E=n E_{1}$ for some integer $n$ and $E<\omega_{2}$ only the lowest energy level is populated. The number of accessible states (assuming, for simplicity, that $g_{1}=1$ for the degeneracy of the lowest energy level) is $\Omega(E)=(n+1)$ and we have $S(E) / E=\left(1 / \omega_{1}\right) n^{-1} \ln (n+1)$. Since $\max \left[n^{-1} \ln (n+1)\right]=\ln 2 \simeq 0.7$ we obtain that [16]

$$
\max (S(E) / E) \simeq \frac{0.7}{\omega_{1}} .
$$

This shows, in particular, that relation (2) should be considered a good estimate for the maximum of $S(E) / E$ rather than just giving an upper bound on $S(E) / E$. It follows that the bound (1) holds only if $R \omega_{1}$ is restricted from below and is violated if $R \omega_{1}$ can be made arbitrarily small. The latter does occur for certain values of the parameter in the boundary condition, as we show in this paper.

In the next section we consider in detail the case of a $(1+1)$-dimensional massless field for which the analysis of the spectrum and the computation of the $\zeta$ function are especially simple. The $(3+1)$-dimensional field is analyzed in Sec. III and the massive field is briefly discussed in Sec. IV. The size of the "forbidden region" in the parameter space is estimated in Sec. V. Section VI contains some concluding remarks.

\section{THE INTERVAL}

We start our analysis with consideration of $(1+1)$ dimensional massless scalar field living on the interval $[0, R]$ with the boundary conditions

$$
\left(\frac{d}{d x} \phi+\frac{h_{0}}{R} \phi\right)_{x=0}=0, \quad\left(\frac{d}{d x} \phi-\frac{h_{1}}{R} \phi\right)_{x=R}=0 .
$$

The energy eigenfunction $\phi_{\omega}(x) e^{\imath \omega t}$ satisfies the differential equation

$$
-\frac{d^{2}}{d x^{2}} \phi_{\omega}(x)=\omega^{2} \phi_{\omega}(x)
$$

and takes the form

$$
\phi_{\omega}(x)=N \sin (\omega x+\delta) .
$$

The spectrum is discrete, $\omega_{n}=\alpha_{n} R^{-1}$, where $\{\alpha\}$ are (positive) roots of the equation

$$
\frac{\tan \alpha}{\alpha}=\frac{h_{0}+h_{1}}{h_{0} h_{1}-\alpha^{2}} .
$$

In general, there may also be bound states (for which $\omega^{2}=$ $\left.-\lambda^{2} R^{-2}<0\right)$ with wave function $\phi_{\lambda}(x)=A e^{(\lambda / R) x}$ $+B e^{-(\lambda / R) x}$. The equation on $\lambda$ is

$$
\frac{\tanh \lambda}{\lambda}=\frac{h_{0}+h_{1}}{\lambda^{2}-h_{0} h_{1}} .
$$
[15]

As a consequence of the Mittag-Leffler theorem, we have

$$
\begin{aligned}
& \left(h_{0} h_{1}-z^{2}\right) \frac{\sin z}{z}-\left(h_{0}+h_{1}\right) \cos z \\
& \quad=\left(h_{0} h_{1}-h_{0}-h_{1}\right) \prod_{\alpha>0}\left(1-\frac{z^{2}}{\alpha^{2}}\right) .
\end{aligned}
$$

This formula helps to evaluate explicitly the sums of inverse powers of the roots. One just has to take the logarithm of both sides of Eq. (9), expand by powers of $z^{2}$, and equate the relevant coefficients. The expressions for any $h_{0}$ and $h_{1}$ are given in [15]. To simplify things, in what follows, we assume that $h_{0}=0, h_{1}=h$. One finds

$$
\begin{aligned}
& \sum_{\alpha} \frac{1}{\alpha^{2}}=\frac{1}{2}-\frac{1}{h}, \\
& \sum_{\alpha} \frac{1}{\alpha^{4}}=\frac{1}{h^{2}}-\frac{2}{3 h}+\frac{1}{6} .
\end{aligned}
$$

Let us consider the case of positive $h$ first. For $h_{0}=0, h_{1}$ $=h$ Eq. (8) becomes

$$
\lambda \tanh \lambda=h
$$

and we see that for positive $h$ there is one bound state [17], $\lambda=\lambda_{b}$. When $h$ is close to zero its value expands in powers of $h$,

$$
\lambda_{b}^{2}=h+\frac{1}{3} h^{2}+\frac{4}{45} h^{3}+O\left(h^{4}\right),
$$


and hence one has

$$
\begin{aligned}
-\frac{1}{\lambda_{b}^{2}} & =-\frac{1}{h}+\frac{1}{3}+O(h), \\
\frac{1}{\lambda_{b}^{4}} & =\frac{1}{h^{2}}-\frac{2}{3 h}+\frac{7}{45}+O(h) .
\end{aligned}
$$

It is important to note that for positive $h$ expressions (10) and (11) obtained by using the Mittag-Leffler theorem include the contribution of the bound state [18]. The small $h$ behavior of (10) and (11) is due to the bound state as one can see by comparing (10), (11), and (13). In particular, this explains why expression (10) is negative when $0<h<2$.

The $\zeta$ function we want to compute is defined for the part of the spectrum with $\omega^{2}>0$. Hence we have to exclude the bound state. Subtracting $1 / \lambda_{b}^{4}$ from (11) we get

$$
R^{-4} \zeta(4) \equiv \sum_{\alpha^{2}>0} \frac{1}{\alpha^{4}}=\frac{1}{80}+\frac{4}{945} h-\frac{1}{675} h^{2}+O\left(h^{3}\right) \text {. }
$$

The divergence of the sum (11) at small $h$ is due to the bound state. After subtraction the sum becomes finite at $h=+0$. In fact, it is bounded for all $h \geqslant 0$, monotonically increasing from $\frac{1}{80}$ at small $h$ to $\frac{1}{6}$ for infinitely large $h$. We conclude that for positive $h$ the function $R^{-4} \zeta(4)<\frac{1}{6}$ and the Bekenstein bound holds perfectly.

Consider now the case of negative $h$. There is no bound state in this case, so that the expression (11) gives us exactly $R^{-4} \zeta(4)$. As a function of $h$ it approaches $\frac{1}{6}$ at $h \rightarrow-\infty$ and grows as $\left(1 / h^{2}-2 / 3 h\right)$ when $h$ is close to -0 . Thus, there is no upper bound for $\zeta(4)$ and the universal entropy bound cannot hold in this case.

It is easy to understand why this happens. The lowest root of Eq. (7), which for $h_{0}=0, h_{1}=h$ reads

$$
\alpha \tan \alpha=-h,
$$

is always between 0 and $\pi / 2$ when $h<0$. For small negative $h$

$$
\alpha_{1}^{2}=-h-\frac{1}{3} h^{2}-\frac{4}{45} h^{3}+O\left(h^{4}\right)
$$

it approaches zero and when $h=0$ it becomes the known zero mode of the Neumann boundary value problem. A higher eigenvalue $\alpha_{n}, n>1$ lies between $(\pi / 2)(n-1)$ and $(\pi / 2) n$ for all values of $h$. The value of $\zeta(4)$ for small $h$ is then mostly due to $\alpha_{1}$. Indeed, approximating

$$
R^{-4} \zeta(4) \simeq \alpha_{1}^{-4}=\frac{1}{h^{2}}-\frac{2}{3 h}+\frac{7}{45}+O(h)
$$

we find agreement with (11) up to $O\left(h^{0}\right)$ terms. The $\zeta(4)$ is unbounded (that indicates a violation of the entropy-toenergy bound) because the lowest energy level $\omega_{1}=\alpha_{1} R^{-1}$ of the spectrum can be made arbitrarily small.
For positive $h$ there is no state with energy close to zero, the lowest $\alpha$ appearing in the interval between $\pi / 2$ and $\pi$. When $h$ passes from the negative to positive values the lowest excited one-particle state (with eigenfrequency $\omega_{1}$ $=\alpha_{1} R^{-1}$ ) becomes the bound state (with $\omega_{b}^{2}=-\lambda_{b}^{2} R^{-2}$ ) and should be excluded. For positive $h$, this saves the entropy bound. However, the possibility to make the lowest energy level arbitrarily small is fatal for the validity of the bound when $h$ is negative.

\section{THE 3D BALL}

In flat $(3+1)$-dimensional space-time consider a massless scalar field confined to a spherical cavity of radius $R$. The boundary condition in this case is

$$
\left(\frac{d}{d r} \phi-\frac{h}{R} \phi\right)_{r=R}=0
$$

where $r$ is the radial coordinate. The condition (17) has the form (3) with $\xi=-(1 / 4) h$ since for the sphere the extrinsic curvature is $K=2 / R$. The Dirichlet boundary condition corresponds to infinite $h$. The energy eigenfunction $\phi_{\omega}$ $=f_{\omega}(r) Y_{l, n}(\theta, \varphi) e^{l \omega t}$ expands in terms of the spherical harmonics $Y_{l, n}(\theta, \varphi)$, the degeneracy being $(2 l+1)$. The equation on the radial function reads

$$
\frac{1}{r^{2}} \partial_{r}\left(r^{2} \partial_{r} f_{\omega}\right)-\frac{l(l+1)}{r^{2}} f_{\omega}=-\omega^{2} f_{\omega} .
$$

Solutions to this equation should satisfy the boundary condition (17) and be regular at $r=0$. There are three types of such solutions.

\section{A. Zero modes}

When $\omega=0$ two solutions are possible, $f_{1}(r)=r^{l}$ and $f_{2}(r)=r^{-l-1}$, only the first being regular at $r=0$. The function $f_{1}(r)$ satisfies the boundary condition (17) only if the parameter $h$ in (17) is some (non-negative) integer, $h=l_{0}$. Then the zero mode exists for $l=l_{0}$ and $f_{1}(r)=r^{l_{0}}$. For $h$ $=0$ this solution is the known zero mode of the Neumann problem. Similar zero modes appear every time when $h$ is a positive integer $l_{0}$. In total, there are $\left(2 l_{0}+1\right)$ of them.

\section{B. Propagating modes}

For $\omega^{2}>0$ the regular solution at $r=0$ is

$$
f_{\omega}(r)=N_{\omega} r^{-1 / 2} J_{l+(1 / 2)}(\omega r),
$$

where $N_{\omega}$ is a normalization constant. Since this solution should satisfy the boundary condition (17) the spectrum is discrete $\omega_{n, l}=\alpha_{n, l} R^{-1}$, where $\left\{\alpha_{n, l}\right\}$ are the roots of the equation

$$
h=l-\alpha \frac{J_{l+(3 / 2)}(\alpha)}{J_{l+(1 / 2)}(\alpha)} .
$$


We have learned from the $(1+1)$-dimensional example considered above that we have to watch for the energy level which may be arbitrarily close to zero. For a fixed $l$ the point $\alpha=0$ is the point where the function staying at the righthand side of Eq. (19) takes the maximal value equal $l$. For negative $h$ the lowest root that may be close to zero is $\alpha_{1,0}$ corresponding to $l=0$. One has $\alpha_{1, l}>2$ for $l \geqslant 1$. When $h$ becomes positive but less than 1 such root appears at $l=1$, $\alpha_{1,1}$. In general, for positive $h$ lying in the interval $l_{0}-1$ $<h<l_{0}$, where $l_{0}$ is positive integer, the lowest root is $\alpha_{1, l_{0}}$ corresponding to $l=l_{0}$. For small $\left(l_{0}-h\right)>0$ one finds

$$
\begin{aligned}
\alpha_{1, l_{0}}^{2}= & 2\left(l_{0}+\frac{3}{2}\right)\left(l_{0}-h\right)-\frac{\left(l_{0}+\frac{3}{2}\right)}{\left(l_{0}+\frac{5}{2}\right)}\left(l_{0}-h\right)^{2} \\
& +\frac{\left(l_{0}+\frac{3}{2}\right)}{\left(l_{0}+\frac{5}{2}\right)^{2}\left(l_{0}+\frac{7}{2}\right)}\left(l_{0}-h\right)^{3}+O\left(l_{0}-h\right)^{4} .
\end{aligned}
$$

\section{Bound states}

These are the regular solutions at $r=0$ with $\omega^{2}<0$,

$$
f_{\lambda}(r)=N_{\lambda} r^{-1 / 2} I_{l+(1 / 2)}\left(\frac{\lambda}{R} r\right),
$$

where $\lambda$ should be determined from the equation

$$
h=l+\lambda \frac{I_{l+(3 / 2)}(\lambda)}{I_{l+(1 / 2)}(\lambda)} .
$$

The function of $\lambda$ staying at the right hand side of Eq. (22) takes at $\lambda=0$ its minimal value equal $l$ and grows monotonically as a linear function for large $\lambda$. The solution to Eq. (22), thus, exists only when $h$ is positive and for a given $l$ there may be no more than one such solution. If $h$ lies in the interval $l_{0} \leqslant h<l_{0}+1$, where $l_{0}$ is a non-negative integer, the solution to the Eq. (22) exists for $l=0, \ldots, l_{0}$. The total number of the bound states (taking into account the degeneracy due to angles) is $\sum_{l=0}^{l_{0}}(2 l+1)=\left(l_{0}+1\right)^{2}$. For small $\left(h-l_{0}\right)>0$ one has

$$
\begin{aligned}
\lambda_{l_{0}}^{2}= & 2\left(l_{0}+\frac{3}{2}\right)\left(h-l_{0}\right)+\frac{\left(l_{0}+\frac{3}{2}\right)}{\left(l_{0}+\frac{5}{2}\right)}\left(h-l_{0}\right)^{2} \\
& +\frac{\left(l_{0}+\frac{3}{2}\right)}{\left(l_{0}+\frac{5}{2}\right)^{2}\left(l+\frac{7}{2}\right)}\left(h-l_{0}\right)^{3}+O\left(h-l_{0}\right)^{4} .
\end{aligned}
$$

We see from Eqs. (20) and (23) that when $h$ passes through the point $h=l_{0}$ the propagating state with the lowest eigenfrequency $\omega_{1}^{2}=R^{-2} \alpha_{1, l_{0}}^{2}$ becomes a bound state with $\omega_{b}^{2}=$ $-R^{-2} \lambda_{l_{0}}^{2}$.

Evaluating sums of inverse powers of the roots we can again employ the Mittag-Leffler theorem. At fixed $l$ one has

$$
z^{-[l+(1 / 2)]} F_{l+(1 / 2)}(z)=\gamma_{l} \prod_{\alpha>0}\left(1-\frac{z^{2}}{\alpha^{2}}\right),
$$

where $\gamma_{l}$ is some constant (see [15]) and $\{\alpha\}$ are the roots (19), $F_{l+(1 / 2)}(\alpha)=0$, where

$$
F_{l+(1 / 2)}(z)=z J_{l+(1 / 2)}^{\prime}(z)+(l-h) J_{l+(1 / 2)}(z) .
$$

Up to terms of order $z^{l+\frac{1}{2}+6}$ it expands as follows:

$$
\begin{aligned}
F_{l+(1 / 2)}(z)= & \frac{z^{l+(1 / 2)}}{2^{l+(1 / 2)} \Gamma\left(l+\frac{3}{2}\right)} \\
& \times\left(1-\frac{1}{4\left(l+\frac{3}{2}\right)} \frac{(l-h+2)}{(l-h)} z^{2}\right. \\
& \left.+\frac{1}{32\left(l+\frac{3}{2}\right)\left(l+\frac{5}{2}\right)} \frac{(l-h+4)}{(l-h)} z^{4}\right) .
\end{aligned}
$$

Taking the logarithm of (24), expanding by powers of $z^{2}$ and using (26) one gets

$$
\begin{aligned}
\sum_{n=1}^{\infty} \frac{1}{\alpha_{n, l}^{4}}= & \frac{1}{16}\left(\frac{1}{\left(l+\frac{3}{2}\right)^{2}} \frac{(l-h+2)^{2}}{(l-h)^{2}}\right. \\
& \left.-\frac{1}{\left(l+\frac{3}{2}\right)\left(l+\frac{5}{2}\right)} \frac{(l-h+4)}{(l-h)}\right)
\end{aligned}
$$

for the sum at fixed $l$. In order to evaluate the $\zeta$ function we have to sum over all possible $l$ and take into account the degeneracy

$$
R^{-4} \zeta(4) \equiv \sum_{l=0}^{\infty}(2 l+1) \sum_{n} \frac{1}{\alpha_{n, l}^{4}} .
$$

We remind the reader that the $\zeta$ function is defined for the spectrum with $\alpha^{2}>0$. The zero modes and bound states are, thus, to be excluded in (28).

When $h$ is negative there are no bound states or zero modes. Substituting (27) into (28) the sum over $l$ can be computed explicitly and is derived in terms of the $\psi$ function as follows 


$$
\begin{aligned}
R^{-4} \zeta(4)= & \frac{2}{3} \frac{(2 h+1)\left(4 h^{2}-5\right)}{(2 h+3)^{3}}+\frac{(2 h+1)}{(2 h+3)^{2}} \Psi(1,-h) \\
& -\frac{16}{(2 h+5)(2 h+3)^{3}}\left[\Psi(-h)-\frac{8}{3}+\gamma+2 \ln 2\right] \\
& -\frac{\pi^{2}}{16} \frac{(2 h-1)^{2}}{(2 h+3)^{2}} .
\end{aligned}
$$

It seems that the function (29) has a pole at $h=-3 / 2$ and $h=-5 / 2$. However, it is easy to check that it is regular at those points. The function (29) monotonically increases from the value $\frac{2}{3}-\pi^{2} / 16$ at $h=-\infty$ (Dirichlet boundary condition) to infinity when $h$ approaches zero. We conclude that the $\zeta$ function is unbounded near $h=0$. The expression (29) is valid also for the positive $h$. However, in this case there may be bound states and zero modes [their appearance signals that (29) has poles at integer $h$ ] the contribution of which must be subtracted from the right-hand side of (29).

When $h$ approaches from below any non-negative integer $l_{0}$ the expression (27) for $l=l_{0}$ grows to infinity. It is due to the fact that the lowest root (20), $\alpha_{1, l_{0}}$, becomes arbitrarily small. Indeed, approximating the sum (27) for $l=l_{0}$ by $1 / \alpha_{1, l_{0}}^{4}$ we find that

$$
\sum_{n=1}^{\infty} \frac{1}{\alpha_{n, l_{0}}^{4}}-\frac{1}{\alpha_{1, l_{0}}^{4}}=\frac{1}{16\left(l_{0}+\frac{5}{2}\right)^{2}\left(l_{0}+\frac{7}{2}\right)}+O\left(h-l_{0}\right) .
$$

Thus, for $h$ less but very close to $l_{0}$ one finds that the $\zeta$ function

$$
R^{-4} \zeta(4)=\frac{\left(2 l_{0}+1\right)}{\alpha_{1, l_{0}}^{4}(h)}+O\left(l_{0}-h\right)^{0},
$$

where $\alpha_{1, l_{0}}^{2}$ is given by Eq. (20), is not bounded from above. This can also be seen from the analysis of the poles in (29).

The whole picture changes when $h$ passes through the point $h=l_{0}$ and becomes slightly greater than $l_{0}$. In this case the root $\alpha_{1, l_{0}}$ disappears. But, instead, there appears a bound state, $\lambda_{l_{0}}^{2}$. In this case, as was explained in the previous section, the expression (27) for $l=l_{0}$ [and, hence, also the righthand side of Eq. (29)] contains the contribution of the bound state which should be excluded [19]. The sum $\Sigma_{n} 1 / \alpha_{n, l_{0}}^{4}$ then is over the propagating modes only and is finite when $h$ approaches $l_{0}$ from above. As a result, the $\zeta$ function (28) is finite when $h \rightarrow l_{0}+0$. A special case is when $h=l_{0}$ exactly. In this case there appears a zero mode $f_{1}=r^{l_{0}}$ which should be excluded when one computes the $\zeta$ function. When $h$ increases, this zero mode becomes the bound state corresponding to $l=l_{0}$ while the rest of the spectrum changes continuously. Therefore, the case $h=l_{0}$ can be achieved by taking the limit $h \rightarrow l_{0}+0$. All of this is repeated every time $h$ becomes close to some positive integer. The general behavior of the spectrum and the $\zeta$ function near every such point is similar to what we had in the $(1+1)$-dimensional case. We conclude that in a way similar to the twodimensional case, the four-dimensional $\zeta(4)$ considered as a function of the parameter $h$ in the boundary condition (17) is not bounded near each point $h=l_{0}$ at which the radial equation (18) has a zero mode.

\section{THE MASSIVE FIELD}

In this section we briefly discuss the massive field. In the presence of mass $m=\mu R^{-1}$ the eigenfrequencies $\omega_{\alpha}$ are defined as $R^{2} \omega_{\alpha}^{2}=\mu^{2}+\alpha^{2}$, where $\{\alpha\}$ are the roots considered in Sec. II. The $\zeta$ function then reads

$$
R^{-4} \zeta(4)=\sum_{\{\alpha\}, \mu^{2}+\alpha^{2}>0} \frac{1}{\left(\mu^{2}+\alpha^{2}\right)^{2}},
$$

where the sum is over states with $\omega_{\alpha}^{2}>0$. We see that due to the mass some of the bound states of the massless field now become the propagating states. In particular, the zero mode is the state (21) with $\lambda=\mu$. The partial summation in (32) for fixed $l$ can again be done with the help of the Mittag-Leffler theorem [15]. After analytical continuation $z \rightarrow \imath \mu$, Eq. (24) reads

$$
\begin{aligned}
\mu^{-[l+(1 / 2)]} \Phi_{l+(1 / 2)}(\mu)= & \gamma_{l} \prod\left(1+\frac{\mu^{2}}{\alpha^{2}}\right), \\
\Phi_{l+(1 / 2)}(\mu)= & \mu I_{l+(1 / 2)}^{\prime}(\mu) \\
& +(l-h) I_{l+(1 / 2)}(\mu) .
\end{aligned}
$$

Taking the logarithm of (33) and differentiating with respect to $\mu^{2}$ one finds

$$
\begin{aligned}
& \sum_{\{\alpha\}, \text { fixed } l} \frac{1}{\left(\mu^{2}+\alpha^{2}\right)^{2}} \\
& \quad=-\frac{d}{d \mu^{2}}\left(\frac{d}{d \mu^{2}} \ln \left[\mu^{-[l+(1 / 2)]} \Phi_{l+(1 / 2)}(\mu)\right]\right) .
\end{aligned}
$$

Of course, using (34) for evaluation of the sum (32) we should watch for the bound states $\left(\mu^{2}+\alpha^{2}<0\right)$ and zero modes $\left(\mu^{2}+\alpha^{2}=0\right)$ and subtract their contribution from the right-hand side of Eq. (34). We have two parameters at our disposal, $h$ and $\mu$. When $h$ is negative there are no states with $\mu^{2}+\alpha^{2} \leqslant 0$. Let us fix $h, l_{0} \leqslant h<l_{0}+1$, where $l_{0}$ is some non-negative integer. In this case, there are $\left(l_{0}+1\right)$ roots $\lambda_{l_{0}}<\cdots<\lambda_{1}<\lambda_{0}$ of Eq. (22),

$$
\Phi_{l+(1 / 2)}(\lambda)=0 .
$$

A zero mode of the massive field appears when $\mu$ equals one of these $\lambda$, say $\mu=\lambda_{k}$. For $\mu$ close to $\lambda_{k}$ one finds that

$$
\Phi_{l+(1 / 2)}(\mu)=C_{k}\left(\mu^{2}-\lambda_{k}^{2}\right)+O\left(\mu-\lambda_{k}\right)^{3},
$$

where $C_{k}$ is some constant.

Substituting this expression into Eq. (34) we find that the sum 


$$
\sum_{\{\alpha\}, \text { fixed } l} \frac{1}{\left(\mu^{2}+\alpha^{2}\right)^{2}}=\frac{1}{\left(\mu^{2}-\lambda_{k}^{2}\right)^{2}}+O\left(\mu-\lambda_{k}\right)^{0}
$$

diverges when $\mu$ approaches $\lambda_{k}$. If $\mu$ is slightly greater than $\lambda_{k}$, the $\lambda_{k}$ state is a propagating state. In fact it is the state with the lowest positive eigenfrequency, $\omega_{1}^{2}=\mu^{2}-\lambda_{k}^{2}$. The expression (35) is divergent because the lowest energy level $\omega_{1}$ can be arbitrarily small. This results in the corresponding divergency of the $\zeta$ function (32).

Now decrease $\mu$ so that it becomes slightly less than $\lambda_{k}$. Then the $\lambda_{k}$ state becomes a bound state of the massive field. Subtracting its contribution from (35) and (34) we get a finite expression. This means that the $\zeta$ function is bounded from above as $\mu \rightarrow \lambda_{k}-0$. This resembles the picture we had in the previous section when parameter $h$ approached a positive integer. In both cases the $\zeta$ function is unbounded in a half vicinity of a point in the parameter space where a zero mode appears. Also, it is so because the lowest eigenfrequency $\omega_{1}$ is arbitrarily small when that point is approached.

\section{THE ESTIMATE FOR THE "FORBIDDEN REGION"}

It follows from the analysis just given that the bound (1) cannot hold for all values of the parameter $h$ in the boundary condition (17). However, since $\zeta(4)$ as a function of $h$ is sharply picked at integer values of $h$, one may expect that the bound (1) holds for almost all values of $h$ except a narrow region near integer $h$. Then (1) would still be valid for a "typical" boundary condition. It is indeed the case. In order to show this we need to estimate the "forbidden region" in the space of boundary conditions where the bound is violated. In order to make the estimate as precise as possible it is best to use the formula (4) and its generalization [16] for arbitrary degeneracy. Using (20) we find for $h$ close to an integer $n$ that

$$
\max (S(E) / E) \simeq R \frac{\ln g_{n}}{\sqrt{(2 n+3)(n-h)}},
$$

where $g_{n}=2 n+2$. The bound (1) is exceeded for $h$ lying in the interval $n-\epsilon_{n} \leqslant h<n$, where for $\epsilon_{n}$ we find using (36) the following estimate:

$$
\epsilon_{n} \simeq \frac{1}{4 \pi^{2}} \frac{\left(\ln g_{n}\right)^{2}}{(2 n+3)} .
$$

In particular, we find that $\epsilon_{0} \simeq 0.00406, \epsilon_{10} \simeq 0.0105$, and $\epsilon_{100} \simeq 0.00352$. We see that the "forbidden region" near each integer $n$ is indeed very narrow and shrinks for large $n$. It follows that the bound (1) holds for almost all negative values of the parameter $h$ except the narrow region $-\epsilon_{0}$ $<h<0$. Recalling the relation between $h$ and the nonminimal coupling $\xi$ in (3), $h=-4 \xi$, we find that the forbidden region for positive $\xi$ is from 0 to $10^{-3}$. Of course, the conformal coupling $\xi=\frac{1}{6}$ is quite far from this region.
For positive $h$ there is a piece of the forbidden region near each integer value of $h$. In order to estimate how dense this region is in the parameter space, let us consider a large interval $0<h<N$. The relevant density then is given by the quantity $(1 / N) \sum_{n=1}^{N} \epsilon_{n}$. Using (37) we find

$$
\frac{1}{N} \sum_{n=1}^{N} \epsilon_{n} \simeq \frac{1}{24 \pi^{2}} \frac{1}{N}(\ln N)^{3}
$$

for large $N$. So, randomly picking a boundary condition with a positive $h$ we almost always put our finger on the right one for which the bound (1) is valid. For example, for the interval from 0 to $N=100$ the probability to choose a wrong condition is $4 \times 10^{-3}$. Thus, the bound (1) still holds for a "typical"' boundary condition.

\section{CONCLUDING REMARKS}

Recall the basic assumptions (see [5]): (i) $S(E)$ is microcanonical entropy defined as the logarithm of the number of accessible states with energy up to $E$; (ii) $E$ is the energy over the vacuum (zero modes are excluded); (iii) all interactions are negligible except the boundary interaction expressed as a boundary condition; (iv) walls of the cavity are ignored. Among these assumptions, the last one is perhaps the most suspicious although we are just following the prescriptions of [5]. From the original idea of Bekenstein (recently restated in [10]) the bound (1) applies to a complete system with $E$ being the total gravitating energy of the system. So it might be that when the boundary condition of the form (3) is imposed the boundary itself may carry some part of the total energy and, possibly, entropy. The bound (5) then should apply to the complete system of the quantum field and walls. This possibility should be further investigated.

Alternatively, one may try to redefine the ground state. Consider for simplicity the two-dimensional field system analyzed in Sec. II. The lowest energy level can be defined as a new vacuum; then the next excited level has energy $\left(\omega_{2}-\omega_{1}\right)$ which is bounded from below for all values of $h$, so that the entropy bound holds in this case. However, the gravitating energy is not something one can define by a random choice of zero. It is not clear at the moment how the gravitating energy is actually computed for the system under consideration. Although it still might be possible, by changing the rules appropriately, to save the bound for all boundary conditions, we prefer to interpret our results, provided the bound applies to the field only, as imposing certain (quite relaxed) constraints on the value of the boundary interactions.

\section{ACKNOWLEDGMENTS}

I would like to thank J.D. Bekenstein, J.S. Dowker, G. 't Hooft and M. Parikh for useful discussions. I thank Bogoliubov Laboratory of Theoretical Physics at JINR, Dubna for hospitality while this work was in progress. 
[1] J. D. Bekenstein, Phys. Rev. D 23, 287 (1981).

[2] J. D. Bekenstein, Phys. Rev. D 30, 1669 (1984).

[3] W. G. Unruh and R. M. Wald, Phys. Rev. D 25, 942 (1982).

[4] J. D. Bekenstein, Phys. Rev. D 49, 1912 (1994).

[5] M. Schiffer and J. D. Bekenstein, Phys. Rev. D 39, 1109 (1989).

[6] J. D. Bekenstein and E. I. Guendelman, Phys. Rev. D 35, 716 (1987).

[7] J. D. Bekenstein, Phys. Rev. D 60, 124010 (1999).

[8] M. Schiffer and J. D. Bekenstein, Int. J. Mod. Phys. C 1, 355 (1990).

[9] D. Page, gr-qc/0005111.

[10] J. D. Bekenstein, gr-qc/0006003.

[11] W. G. Unruh, Phys. Rev. D 42, 3596 (1990).

[12] M. Schiffer and J. D. Bekenstein, Phys. Rev. D 42, 3598 (1990).

[13] A. O. Barvinsky and S. N. Solodukhin, Nucl. Phys. B479, 305 (1996)
[14] I. Moss, Class. Quantum Grav. 6, 759 (1989).

[15] J. S. Dowker, Class. Quantum Grav. 13, 585 (1996).

[16] For arbitrary degeneracy $g_{1}>1$ we find that $\max (S(E) / E)$ $=\ln \left(g_{1}+1\right) / \omega_{1}$.

[17] In general, there is one bound state if the following conditions are satisfied: $-h_{0} h_{1} \geqslant 0, h_{0}+h_{1} \geqslant 0$, and $h_{0}+h_{1} /-h_{0} h_{1} \geqslant 1$; two bound states if $h_{0}+h_{1}>0$ and $-h_{0} h_{1}<0$, and no bound states in any other case.

[18] It is particularly easy to see for a special case $h_{0}+h_{1}=0$. The bound state then is $\lambda_{b}^{2}=-h_{0} h_{1}$ and the roots of Eq. (7) are $\alpha_{n}=\pi n, n=1,2, \ldots$. Including both the bound state and the states with $\alpha^{2}>0$ it is straightforward to evaluate the sum $-1 / \lambda_{b}^{2}+\sum_{n=1}^{\infty} 1 / \alpha_{n}^{2}=1 / h_{0} h_{1}+\frac{1}{6}$. It exactly coincides with what we get by expanding the logarithm of Eq. (9) by powers of $z^{2}$. The contribution of the bound state is evident. I thank J.S. Dowker for this example.

[19] One should also subtract the contributions of all other bound states appearing for $l=0, \ldots, l_{0}-1$. 